\title{
Evolución de una crisis epiléptica generalizada, no tratada: Convulsiones benignas del desarrollo
}

\author{
Dr. Marcelo Devilat B. ${ }^{1,2}$; Dr. Cat los Acevedo Sclt ${ }^{3}$; Dr. Rodrigo Chamorro $0 .{ }^{1}$
}

First generalized non treated epileptic seizure: Prognosis

The first epileptic crisis of a child force physicians to therapeutic decissions involving knowledge of its evolution and prognosis. This study was aimed to delineate prognosis, throughout an at least three years long follow up, in 50 chilaren after their first non provoked nor treated generalized epileptic crisis, all free from known predisposing factors for recurrence. Eleven $(22 \%)$ patients had a second seizure, $9(81 \%)$ within the following 2 years after the first episode. Epilepsy among second grade relatives, age, sex, type of generalized crisis and previous morbility were not significant varjables for recurrence $(p>0.05)$. The scoond sezure was similar to the first one in 1:2 patients. This report demonstrates that few recurrences can be expected in children with a first non treated epileptic seizure. if the characteristics of every patient ate properly considered.

(Key words: epilepsy, first crisis, generalized, rate of recurrence, time of recurrence, pred isposing factors, untreated.)

Cuando un niño presenta epilepsia, la indicación de iniciar un tratamiento con anticonvulsivantes es perentoria, pero la situación es diferen. te si el enfermo ha tenido sólo una convulsión. Esto último plantea numerosas interrogantes acerca de la conducta a seguir ante una primera crisis epiléptica, razón por la cual las opiniones

1. Servicio de Neurología y Psiquiatria Infantil. Hospital Luis Calvo Mackenna.

2. División Ciencias Médicas Oriente, Facultad de Medicina. Universidad de Chile.

3. Departamento de Neurología Infantil. Hospital San Juan de Dios, Facultad de Medicina. Universidad de Chile. al respecto están divididas ${ }^{1-9}$ y generalmente basadas en la tradición clínica. Por otra parte, últimamente ha aparecido la ide ${ }^{10}$ de posponer el tratamiento en aquellos niffos portadores de "convulsiones benignas del desarrollo" o "epilepsia benigna de la infancia", cuyas características clínicas pueden predecir un buen pronóstico.

E1 conociniento del curso que adopta una primera crisis epiléptica no provocada y de los factores relacionados con las recurrencias, es esencial para sugerir un plan terapéutico racio$\mathrm{nal}^{11}$. A pesar de la importancia del tema, sólo en los últimos años se han emprendido investigaciones metodológicamente bien estructuradas para abordar el problema en adultos ${ }^{11,12}$ y en 
niños ${ }^{11,13-15}$, habiéndose descrito además aque. llas variables que son significativas para predecir las recurrencias ${ }^{11-15}$.

El objetivo de esta comunicación es determinar el riesgo y la oportunidad de las recaidas, en 50 niños que presentaron una primera crisis epiléptica generalizada, no provocada y no tratada, quienes fueron seguidos al menos por 3 años. Asimismo, se analizarán los factores predictivos de recurrencia, excluyendo a aquellos gue son significativos en la literatura. Por último, se describirán las caracterjsticas clínicas de las crisis con las que recurren los enfermos.

\section{MATERIAL Y METODO}

F.n una publicación anterior se describieron las características de la presenta muestra y la metodología empleada ${ }^{16}$.

Ochenta $y$ ocho niños ingresaron al estudio hasta mayo de 1983 . Fn todos se registró el electroencefalograna (LEG) poco después de la primera crisis, en vigilia o sueño, con hiperventilación o fotoestimulación -según la edad- en el momento del examen. Al finalizar el período de observación, 50 pacientes cumplian con el requisito de haber sido controlados al menos por 36 meses ( $\bar{x} 39,84$, rango 36 a $\$ 4$ meses). Estos últimos enfermos constituyen el material de esta publicación.

Veintiocho eran hombres, sólo ocho tenían antecedentes de epilepsia en familiares de segundo grado, seis riños habían terido alguna alteración perinatal, uno estaba en tratamiento prolongado con prednisona, uno padecia del sindrome de Goldentart y 42 no tenjan antecedentes mórbidos. Veintiún enfermos presentaron un gran mal tónico-clónico generalizado, 18 crisis tónica generalizađa y 11 crisis atónicas. La edad promedio de la primera crisis fue de 6,43 (DE 4,34), rango 0,5 a 15 años. En ocho pacientes ésta ocurrió antes de los 2 años de edad. En el EEG un niño presontó lentitud de la actiridad basal, 6 actividad paroxística lenta generalizada y en los demás el EFG era normal.

\section{RESULTADOS}

Treinta y nueve de los 50 enfermos ( $78 \%$ ) estuvieron libres de crisis durante el tiempo de control. En $11(22 \%)$, acurrió un segundo episodio a $\operatorname{los} 4$ ( 2 pacientes), 5 y 6 ( 2 niños en cada caso), 10,11,17,19, 30 y 34 (un niño en cada caso) meses después de la primera crisis. El sexo era masculino en 7 casos de recurrencias. Tres pacientes tenian antecedentes de epilepsia en familiares de $2^{\circ}$ grado y 8 no. El primer episodio había sido en 4 enfermos gran mal, en 6 , ataques tónicos y en 1, episodios atónicos. Un paciente, cuya primera crisis aconteció antes de $\operatorname{los} 2$ años de vida y 10 en los que se presentó después de esa edad, tuvieron un segundo episodio crítico. No se detectaron diferencias estadisticamente significativas (Chi cuadrado y prueba de Fisher, $\mathrm{p}>0,05$ ) de sexo, edad, antecedentes familiares y tipo de la crisis entre pacientes con y sin recu. trencias.

De 4 pacientes que presentaron gran mal en su primera crisis y recurrieron, en 3 el segundo episodio fue similar al primero, $y$ en uno fue focal. De $\operatorname{los} 6$ enfermos con crisis tónicas, que recayeron, 3 recurrieron con el mismo tipo de crisis, en 2 el segundo episodio fue gran mal y en otro focal. Sólo un niño cuya primera crisis fue atónica recurrió, esta vez, con un episodio de tipo focal. En síntesis, 6/11 pacientes $(54,54 \%)$ recurrieron con crisis similares a la primera y $5(45,45 \%)$ con tipos diferentes.

\section{DISCUSION}

Es dificil comparar estos resultados con los de otras series (ver tabla) por cuanto en ellas los pacientes presentaban factores de rjesgo que se excluyeron en este grupo ${ }^{16}$. En algunas publicaciones las cifras son semejantes a las que of rece$\operatorname{mos}^{9,} 11,13$; en otras son diferentes ${ }^{14}, 15 . \mathbf{L a}$ diversidad de resultados está en relación con métodos de estudio y características de las muestras no comparables entre sí. No obstante, en niños con crisis generalizadas o focales secundariamente generalizadas con examen neurológico y EEG normales, el porcentaje estimado de recurrencias es muy similar al de este estudio ${ }^{14}$.

El momento en que ocurren las recaídas depende de la duración del período de observación. Al firal del primer affo de control se habian acumulado $12,8 \%$ de recurrencias; al tercer año o más, 22\%. Lo importante, sin embargo, es cuando sucede la mayor cantidad de recurrencias, por el valor pronóstico que esto involucra. La mayoria de las recaidas se observaron dentro de los 2 años después de la primera crisis, hecho que acurre tanto en niños ${ }^{11}, 14,15$ como en adul$\operatorname{tos}^{12}$. Esto también ha sido visto en pacientes en tratamiento por epilepsia ${ }^{17}$ y en aquellos en que la terapia se suspende ${ }^{18}, 19$. Lo anterior es relevante, pues determina el tiempo de observación a que deben someterse los enfermos.

Los factores con significación estadística que predicen recurrencias en niños con crisis epilép- 
Tabla

Recurrencia de prinera crisis epiléptica generalizada en nithos

\begin{tabular}{|c|c|c|c|c|c|}
\hline & \multirow[t]{2}{*}{ Año } & \multirow{2}{*}{$\begin{array}{l}\text { Períndo de } \\
\text { observación } \\
\text { (Meses) }\end{array}$} & \multirow{2}{*}{$\begin{array}{c}\text { Pacientes } \\
\text { tratados } \\
\%\end{array}$} & \multicolumn{2}{|c|}{ Recurrencias } \\
\hline & & & & $\mathrm{n}$ & $\%$ \\
\hline Thotnas & 1958 & - & 100 & $13 / 48$ & 27 \\
\hline Hauser y cols. & 1982 & 36 & 69 & $20 / 101$ & 19,80 \\
\hline Hirtz y cols. & 1984 & $24 \quad-$ & 27 & $152 / 315$ & 48,25 \\
\hline Canifield y cols. & 1984 & $32,4(\bar{x})$ & 52 & $33 / 75$ & 44 \\
\hline Camfjeld y cols. & 1984 & $32,4(\bar{x})$ & 52 & - & 30 \\
\hline Cantield y cols. & 1985 & $12-36 \quad$ (t) & 100 & $4 / 27$ & 14,81 \\
\hline Devilat y cols. & 1987 & $36-56$ & 0 & $11 / 50$ & 22 \\
\hline
\end{tabular}

$\bar{x}=$ promedio.

$\tau=$ rango .

ticas únicas, generalizadas o parciales, son variados: antecedente de epilepsia en familiares de primer grado ${ }^{11}$, convulsiones neonatales ${ }^{15}$, crisis sintomáticas ${ }^{11-15}$, crisis parciales ${ }^{13-15}$, examen neurológico anormal ${ }^{14}$ y EEG específico para epilepsia ${ }^{11,14}$. Otras variables estudiadas por nosotros, algunas como tipo de crisis generalizada no incluidas en la literatura, no demostraron ser factores predictores de recurrencias. En conse. cuencia, se puede demostrar que $22 \%$ de recaidas de esta investigación no está sesgada por varjables de importancia $y$, por lo tanto, es una cifra que tiende a acercarse a la realidad.

De nuestros resultados se puede inferir que uno de cada dos enfermos $(54,5 \%)$ que recae, to hará con un tipo de crisis similar a la primera, hecho importante, pues puede contribuir a disminuir la ansiedad que provoca en los padres un segundo episodio epiléptico. La mitad de los pacientes recurren con una crisis diferente a ta primera: no se sabe por qué y en la literatura no hay referencias al respecto. Es posible que factores maduracionales del SNC estén involucrados, pero es difícil probarlo, ya que $98 \%$ de los enfermos tenía ritmo basal normal en el EEG.

Ninguno de los pacientes recibió tratamiento anticonvulsivante, hecho que $\mathrm{da}$ a la presente muestra un carácter distintivo respecto al resto de las publicaciones ${ }^{11,13-15,20}$, con una excepción, donde se mezclaron niños con crisis ocasio. nales y crisis no provocadas'. Nuestra investigación, considerando lo anterior, deberia haber demostrado cifras de recurrencia superiores; sin embargo, se ha probado que el tratamiento no influye en las recaidas ${ }^{11,} 15,19$, por lo que se puede deducir que serán los factores anteriormente aludidos los que definirán el pronóstico. Lo an- terjor destaca la importancia de una correcta evaluación de cada paciente, antes de recomendarle iniciar o no un tratamiento.

Esta investigación proporciona dos hechos de interés que no han sido enfatizados en la literatura: que 1 de cada 4 niños con una crisis epiléptica generalizada, no tratada y sin factores significativos de recurrencias, vuelve a presentar un segundo episodio y que éste es en el $50 \%$ de los casos similar al primero. Se confirma, además, que las recurrencias son precoces y que pocas recaidas se pueden esperar si el enfermo está libre de crisis por 2 ó 3 años. Esto último plantea un probtema adicional si un paciente presenta un segundo episodio epiléptico, uno o dos años después del primero: ¿debería recibir tratamiento o habría que esperar una tercera crisis? La interrogante conduce al tema de la oligoepilepsia $^{21}$, para el cual tampoco existe una respuesta definitiva. Futuras investigaciones deberian contribuir a resolver el problema.

Por ültimo, es necesario destacar que, con algunas pequeftas diferencias, los nifros de la investigación que se comenta, parecen ser portadores de lo que se ha llamado "convulsiones benignas del desarrollo"10 y que se ha logrado identificar un grupo de pacientes que no necesitan tratamiento, de acuerdo a la proposición de Freeman y cols. ${ }^{10}$, bajo la premisa que el "remedio puede ser peor que la enfermedad"22.

\section{RESUMEN}

La presentación de una primera crisis epiléptica en un niño obliga al médico a tomat una decisión terapéutica, para lo cual se debe conocer 
la evolución y pronóstico de ella. En esta investigación se ha intentado responder a dicha interrogante, a través del seguimiento de al menos 3 años, de 50 niños que presentaron una primeta crisis epiléptica generalizada, no provocada, no tratada y sin los factores significativos de recurrencia, comunicados en la literatura. E1 78\% de los enfermos no recayó y el $81 \%$ de los que volvieron a presentar crisis lo hicieron dentro de los 2 años siguientes del primer episodio. Epilepsia en familiares de $2^{\circ}$ grado, edad, sexo, tipo de crisis generalizada y morbilidad previa no fueron variables significativas de recurrencias. Uno de cada 2 enfermos que recae lo hace con una crisis similar a la primera. Esta comunicación demuestra que pocas recurrencias se pueden esperar en niños con una primera crisis epiléptica no tratada si se considera adecuadamente las características de cada paciente.

\section{REFERENCLAS}

1. Janz D.. Indikationen zur Finleintung und Beendigung einer antiepileptischen Therapie bei Jungendlichen und Erwachsenenen. In: Kruse R., Hrsg: Epilepsie, Stuttgart, Germany: Thieme, 1971;15-19.

2. Degen R.: Die Kindlichen Anfallsleiden. Epileptische und nichtepileptische Anfälle, Stuttgart, Germany: Hippokrates, 1976;69.71.

3. Matthes A.: Epilepsie. Diagnostik und Therapie für Klinik und Praxis, Stuttgart, Germany: Thieme, $1977 ; 82$.

4. Penrose R, Wilder J.B.: Epilepsy, Philadelphia: F. A. Davis, 1968; 177-178.

5. Weber M. Loisseau P.: Epilepsies et epileptiques, Paris: Ligue Ftançaise contre l'epilepsie, 1980.

6. Janz $D$.: Problems ecountered in the treatment of epilepsy. In: Birkmayer W., ed.: Epileptic seizuresbehaviour-pain. Viena: Hans Huber, 1976;65-75.

7. Doose H.: Indikationen zur Einleitung im Kinde- salter. In: Kruse R., Hrsg.: Epilepsie, Stuttgart, Gemany: Thieme, 1971;9-15.

8. Reynolds $E H$ : Drug treatment of epilepsy. In: Jones O.M., ed,: Epilepsy 79, London 79, London: The Brithish Epilepsy Association, 1979: 31-42.

9. Thomes M.H.: Single seizure-its study and management. JAMA 1959;169:457-459.

10. Freeman J.M. Tibbles J., Camfietd C., Camfield P.: Benign epilepsy of childhood: a speculation and its tamifications. Pediatrics 1987; 79: 864-868.

11. Hauser W.A., Anderson V.E., Loewenson R.B. MCRoberts S.M.: Seizure recurtence after a first unprovoked seizure. New Engl J Med 1982; 307 : $522-528$.

12. Cleland P.G. Mosquera I., Steward W.P., Foster J.B.: Prognosis of isolated seizure in adult life. Brit Med J 1981; 283: 1364.

13. Camfield P.R., Camfield C.S., Smith E.C., Tibbles $J . A R$.: Newly treated childhood epilepsy: a psospective study of recurrences and side effects. Neurology 1985: 35: 722-725.

14. Camfield P.R., Camfield C.S, Dooley J.M., Tibbles J.A.R., Fung $T$., Gamer B.: Recurrence after a first unprovoked afebrile seizure in childhood. Ann Neurol 1984; 16: 379 .

15. Hirtz D.G., Ellenberg J.H., Netson K.B.: The risk of a recurrence of nonfebrile seizures in children. Neurology 1984; 34: 637.641.

16. Devilat M. Menéndez P., Chamorro R., Acevedo C. Seguimiento de niños con una crisis cpiléptica no tratada. Rev Chil Pediatr 1984; 55: 9-10.

17. Shorvon S.D., Reynolds E.H.: Early prognosis of epilepsy. Brit Med J 1982; 285: 1699-1701.

18. Devilat M. Valenzuela B., Bhtumel J.E.: Riesgos de recaídas en niñ̃os con epilepsia después de suspender medicamentos anticonvulsivos. Rev Chi Pediats 1987; $58: 29-33$.

19. Devilot M., Germain L., Valenzuela B.: Pronóstico a largo plazo en niffos con crisis eptlópticas tratadas por 5 años. Rev Chil Pedialr 1983; 54: 319-324.

20. Blom S., Heijbel J., Bergfors P.G.: lncidence of epilepsy in children: follow-up study three years after the first seizure. Epilepsia 1978; 19: 343-350.

21. Devilat M. Menéndez P.: La oligoepilepsia. Una manera diferente de ser epiléptico? Rev Chil Pediatr $1985 ; 56: 53-56$.

22. Reynolds E.H.: Chronic antiepileptic toxicity. Epilepsia 1975; 16: 319-352. 